\title{
The ideal approach of ovarian dermoid cyst excision; the predicament of laparoscopy versus laparotomy
}

\begin{abstract}
Purpose: The aim of the current retrospective study for reviewing the cases of dermoid cyst managed at our hospital during the study period, to find out the best and safest management approach after taking in consideration the related factors.
\end{abstract}

Setting: This analysis took place at the department of Gynecology and Obstetrics at Jordan University Hospital.

Material and methods: The retrospective records were reviewed for over 6 years (from January 2015 to January 2021). The sample of study included a total number of 87 patients operated with ovarian dermoid. Thirty-five patients $(40.22 \%)$ presented with abdominal mass, 16 patients (18.39\%) were accidently found to have this kind of ovarian cysts, 12 patients $(13.79 \%)$ presented with pressure symptoms, 11 patients $(12.64 \%)$ presented with dyspareunia and dysmenorrhea, 8 patients $(09.19 \%)$ with abdominal pain, and 5 patients $(05.74 \%)$ with recurrent attacks of nausea. Thirty-nine patients $(44.83 \%)$ treated by laparotomy, another 39 patients $(44.83 \%)$, the management procedure completed laparoscopically, and 9 patients $(10.34 \%)$, the procedure started laparoscopically, then converted to laparotomy. Sixty-one patients $(70.11 \%)$ were found to have right sided ovarian dermoid, while 19 patients $(21.84 \%$ ) were found to have bilateral, and 7 patients $(08.05 \%)$ were found to have left sided one. The size of the cyst was more than $11 \mathrm{~cm}$ in 40 patients (45.98\%), while less than $5 \mathrm{~cm}$ in 5 patients $(5.75 \%)$.

Results: ovarian cystectomy performed in 70 patients $(80.46 \%)$, and salpingooophorectomy on 17 patients (19.54\%) on both reproductive and postmenopausal group. The average blood loss and operative time were more on the laparoscopic group; $298.7 \mathrm{ml}$, 98.8 minutes respectively.

Conclusion: Laparotomy and ovarian cystectomy seems to be superior to other methods of management for ovarian dermoid, particularly when the size of the cyst is more than $5 \mathrm{~cm}$, and predominantly the cysts presentation bilaterally.

Keywords: ovarian dermoid, laparotomy, abdominal pain
Volume 12 Issue 4 - 202I

\author{
Kamil M Fram, Shawqi S Saleh, Nadia A \\ Muhaidat, Farah K Fram, Rand K Fram, \\ Abdallah I Massad, Rana M Haddad, Zaid H \\ Sunna, Eiman Sadaqa \\ Department of Obstetrics and Gynecology, Jordan University \\ Hospital,Amman, Jordan
}

\section{Correspondence: Kamil Mosa Fram, MD, Professor of Obstetrics and Gynecology, School of Medicine, The University of Jordan, Amman, Jordan, Tel ++962 795642000 ,}

Fax++962 6553 8845, Email kamilfram@gmail.com

Received: June 24, 2021 | Published: July 07, 202 I

\section{Introduction}

It is well known through history of gynecological science that dermoid ovarian masses are the most common germinative ovarian tumors, affecting the women in young age groups during the golden reproductive years, with a mean age of thirties, whereas on the other side of epithelial neoplasm with the potential of malignancy affects older age group with a mean age of fifty-five years. ${ }^{1-3}$ Fortunately, among the female population during their reproductive years, dermoid tumors exhibit a slow growth rate, with an average of $1.8 \mathrm{~mm}$ per year, which has led some physicians to embrace nonsurgical approach for the management of smaller tumors (less than $5 \mathrm{~cm}$ in diameter); the conservative approach reported to be located on the top of the list. ${ }^{4}$ Generally speaking, dermoid tumors are weird and wonderful; diverse and different, and being of a totipotential germ cell origin they have the ability to develop into many types of mature tissues namely hair and teeth ${ }^{5}$ as well as they present in bilateral location in $10 \%$ of cases. ${ }^{6,7}$ Adding to their diversity upon presentation, they may vary in size from minute tumors to large ones filling the whole of the peritoneal space. ${ }^{8,9}$ Despite the painful truth, in affecting a large population spanning women form all age groups, dermoid cysts rarely present the physician with either a diagnostic or therapeutic issue.
Nevertheless, complications that may arise take various forms and may have a long term effect on the patient, thus posing a predicament for the diagnostician. ${ }^{4,9}$

Taking into consideration their germ cell origin, dermoid tumors can arise as early as at birth, and may be inconspicuous for the whole life of the female until accidentally diagnosed or a complication arises such as rupture or pressure symptoms from large size growth. The clinical presentation of such tumors varies widely form dull ache in the lower back and thighs, to abnormal vaginal bleeding or pressure symptoms such as urinary or bowel disturbances. Kept in mind, caution coupled with caution of the classical and non-classical presentations, the acute surgical pain due to compromise to the ovarian blood supply (ovarian torsion) caused by tumors large enough to twist the ovarian pedicle is a serious emergency that need a prompt intervention. ${ }^{10}$ In spite of their common benign behavior, one should keep in mind that dermoid tumors can exhibit an unpredictable course and progress to cancer in $1-3 \%$ of cases..$^{11,12}$ Although the exact cause of dermoid tumors is still a topic of research, their origin, the germ cell, is a normal cell type that can differentiate normally to any mature cell type, such as previously stated to teeth, skin, hair, eyes, fat and glands. ${ }^{13}$ This fact creates some pressure on the physicians whom take over to treat such disease, and puts the therapist in a whirlwind. 
The objective of this study is to demonstrate our experience in identifying and managing dermoid tumors with more attention paid to cases affecting young age groups. Our experience has given us the ability to deal with these cases in a professional manner, especially with the harmony of the treating medical team, with a continuous development in the level of positive and promising results.

\section{Patients and methods}

During the interval between the first of January 2015 and the first of January 2021, a total of 87 patients, were identified to have dermoid tumors and considered to be suited for surgery. These patients were surveyed for the aim of this study. This study was conducted after the approval conducted by the Institutional Review Board (IRB), the Ethics committee and the Scientific Research Committee (SRC) at our hospital. We used the SPSS (Statistical Package for the Social Sciences) for data analysis. Preoperative assessment including full detailed clinical history, physical examination, routine laboratory tests, chest X-ray was performed for all patients. In particular cases, according to the presentation, age group and possibilities of the nature of the adnexal masses, a CT scan, MRI and plain abdomen imaging were ordered and done for some patients. The tumor markers, in particular CA19.9, CA125 and AFP (alpha fetoprotein) were obtained for most patients when the suspension of possible malignancy took place and being in the front thinkable. Thorough counselling about the nature of surgery, different possibilities and complications encompassing simple cystectomy and possibility of radical surgery based on the nature of the cyst which was almost was planned and determined for all patients preoperatively. Our advice based mainly on our experience coupled with the operative findings. All patients counselled thoroughly, and all questions that revolve around their minds and disturb them and/ or hinder their decision, were addressed and clarified. In emergency situations, regardless the approach of management, or when patients presenting with ruptures cysts, or whom are considered a candidate for conservative management; all were eliminated from this study.

In our country Jordan, much efforts were paid to all patients' inquiries and those of their families regarding the prospects of future fertility and aspects of feminine life were addressed in intricate details. All 87 patients surveyed in this study were diagnosed to have either unilateral or bilateral dermoid cysts. Specimens excised in all cases were sent for histopathological examination and for the definite verification for the diagnosis of dermoid cyst. After the decision for surgical excision was taken, the dilemma for the best approach jumps to front of mind; the 3 holes' standard laparoscopic technique versus the standard open laparotomy procedure which was performed via an abdominal wall incision in layers. Taking peritoneal wash for cytology on both situations was the standard initial step. Intraoperatively, when a torsion of the ovarian pedicle was noted, appropriate stepladders were followed to regain the viability of the ovarian tissue in systemic, optimal organized manner including immediate de-torsion and covering the ovarian tissue with an abdominal gauze soaked with warm hot saline. In most cases, the operation of choice was an attempt of cystectomy to preserve the ovarian tissue; a scalpel blade number 12 is used to smudge a fine temperate scar on the wall of the cyst, a skillful art done perfectly, gained by experience and proper practice, efforts paid consuming the time needed without any form of rush, followed by avaricious the edges of the cyst by fine mosquitoes instruments and slow blunt traction with fingers apart to undress the cyst capsule and deliver the dermoid cyst intact out of its bed.
Eventually, the cysts were excised more easily in the course of the deliberate unfastened procedures, as well as the supreme homeostasis secured for any bleeding spots that the surgeon came across during surgery. Willfully, this process was more difficult in the laparoscopic group as the sense of rupture being a scenario impose its presence, specifically in the presence of adhesions, which is not uncommon in these cases. Keeping in mind the nature of the dermoid cyst various tissue component and thick capsule, blunt dissection and excising it form the ovarian tissue more feasible and easier in the open approach, and rupture would seldom occur. Perhaps the fear and obsession with the explosion of the cyst inside the abdomen, makes us from the traditional conservative party to deal with these cases with a great degree of satisfaction. The cysts would send intraoperatively for frozen section reporting to look for risk of malignant transformation, together with resection of necrotic ovarian tissue. Once the pathologist sends back a reply that the cyst is of benign nature, closure of the abdominal wall with steps and in layers is carried out.

\section{Results}

For the purpose of this study, which was suggested, designed, and sanctioned by the authors to carry on this study. The medical files of all patients who were diagnosed to have an ovarian dermoid tumor and underwent surgical excision, and having the inclusion criteria were reviewed in a retrograde manner. The total number of patients who were candidates for review during the interval spanning form January 2015 to January 2021 was 87 patients. At the time of surgical excision, mean age of the patients was 32.7 years (range 16-53); mean gravidity among married patients was 1.79 (range $0-8$ ), whereas the mean parity was 1.43 (range $0-4$ ). Upon presentation, 75 patients were married while 12 patients were single. Patients reviewed had a mean weight of $66.6 \mathrm{~kg}$ (range 46-90) as illustrated in Table 1. The presenting complaint in these patients was an abdominal mass in $35(40.22 \%)$ patients, accidental finding in 16 patients $(18.39 \%)$, pressure symptoms, dyspareunia and dysmenorrhea in 12,11 patients respectively $(13.79 \%, 12.46 \%)$ as illustrated in Table 2 . In 61 patients (70.11\%) of cases were found to have right sided cysts, while 19 patients $(21.84 \%)$ were identified to have a left sided one, and a larger cyst more than $21 \mathrm{~cm}$ on 21 patients $(24.14 \%)$, part of them were identified accidentally as seen in Table 3 . It was noted at the time of surgery that cysts were twisted 4 times in 4 cases, 3 times in $1 \mathrm{case}$, and once in $1 \mathrm{case}$. The size of the cysts was $11-20 \mathrm{~cm}$ in 29 patients $(33.33 \%), 6-10 \mathrm{~cm}$ in 27 patients $(31.03 \%)$ as illustrated in Table 4. Laparotomy was the main approach for surgical excision, as 62 patients $(71.25 \%)$ underwent this procedure form the start, while another 14pateints $(16.09 \%)$ started with laparoscopic surgery and converted to the laparotomy group as seen on Table 5 .

Table I Demographic data of the patients

\begin{tabular}{lll}
\hline Variable & Range & Mean \\
\hline Age (years) & $16-58$ & 35.7 \\
Gravidity (G) & $0-8$ & 1.8 \\
Parity (P) & $0-5$ & 1.4 \\
Weight (Kg) & $46-90$ & 66.6 \\
\hline
\end{tabular}


Table 2 Chief complaint

\begin{tabular}{lll}
\hline Variable & Number & Percentage (\%) \\
\hline Abdominal mass & 35 & 40.22 \\
Accidental & 16 & 18.39 \\
Pressure symptoms & 12 & 13.79 \\
Dyspareunia/ dysmenorrhea & 11 & 12.64 \\
Abdominal pain & 8 & 9.19 \\
Nausea & 5 & 5.74 \\
Total & 87 & 100 \\
\hline
\end{tabular}

Table 3 Dermoid presentation

\begin{tabular}{lll}
\hline Variable & Number & Percentage \% \\
\hline Right side & 61 & 70.11 \\
Left side & 19 & 21.84 \\
Bilateral & 7 & 8.05 \\
Total & 84 & 100 \\
\hline
\end{tabular}

Table 4 Size of the dermoid

\begin{tabular}{lll}
\hline Variable $\mathbf{( c m )}$ & Number & Percentage (\%) \\
\hline$\leq 5$ & 10 & 11.5 \\
10-Jun & 27 & 31.03 \\
$20-$ Nov & 29 & 33.33 \\
$\geq 21$ & 21 & 24.14 \\
Total & 87 & 100
\end{tabular}

Table 5 Surgical procedure

\begin{tabular}{lll}
\hline Variable & Number & Percentage (\%) \\
\hline Laparotomy & 62 & 71.26 \\
Laparoscopy converted laparotomy & 14 & 16.1 \\
Laparoscopy & 11 & 12.64 \\
Total & 23 & 100 \\
\hline
\end{tabular}

In accordance with the patients' age, fertility and presenting symptoms, different measures and surgical procedures were undertaken to manage the cysts. Seventy cysts $(80.46 \%)$ were excised by ovarian cystectomy and enucleation. seventeen patients (19.51\%) underwent salpingo-oophorectomy in both reproductive and postmenopausal age groups, 1 patient $(1.15 \%)$ underwent right salpingo-oophorectomy, and 1 patients underwent left salpingo-oophorectomy, where the histopathological examination in the latter two cases established the presence of necrotic ovarian tissue due to torsion as seen in Table 6. In certain cases there was an accompanying surgical procedure that included myomectomy in 12 cases $(13.79 \%)$, total abdominal hysterectomy in 11 cases $(12.64 \%)$ as illustrated in Table 7 . All cysts were removed intact with no spillage of cysts content reported and with healthy ovarian tissue preserved.

Table 6 Surgical procedure

\begin{tabular}{lll}
\hline Variable & Number & Percentage (\%) \\
\hline Ovarian Cystectomy & 70 & 80.46 \\
Salpingo-oophorectomy * & 10 & 11.49 \\
Salpingo-oophorectomy \# & 7 & 8.05 \\
Total & 87 & 100 \\
\hline
\end{tabular}

*, reproductive age group; \#, postmenopausal age group

Table 7 Concomitant surgical procedure

\begin{tabular}{lll}
\hline Variable & Number & Percentage (\%) \\
\hline Myomectomy & 12 & 13.79 \\
T.A.H* & 11 & 12.64 \\
Adhesiolysis & 5 & 5.74 \\
Omentectomy & 3 & 3.44 \\
Cesarean section & 2 & 2.3
\end{tabular}

\section{*,T.A.H; total abdominal hysterectomy}

In fact, it was noted that the mean operative time for uncomplicated laparotomy ovarian cystectomy without accompanying surgical procedure was 75 minutes (range 45-120 minutes), while on the other hand, in the laparoscopic group, the mean operative time was 198 minutes (range 90-120 minutes) as seen in Table 8. This discrepancy in operative time between the two groups was in part due to the time dedicated during laparoscopic surgery for irrigation of the pelvis to make certain of pelvic clean out. As soon as the cyst was excised, it was sent for frozen section analysis straightaway, to verify the benign nature of the cyst and rule out possibility of malignancy to determine the need for further surgical procedures. Pathological verification of the definite mature cystic teratoma without atypia was quite clear in all cases. No complications during surgery occurred. On average, the hospitalization time was 3.48 days (range 2-7). During follow up visits and postoperative care, no complications were reported with follow up period which ranged from 2-24 months (mean 12.13). The pregnancy was confirmed in 11 patients after surgery.

Table 8 Operative surgical variables

\begin{tabular}{lllll}
\hline Variable & Average laparotomy & Mean & Average laparoscopy & Mean \\
\hline Blood loss $(\mathrm{ml})$ & $100-750$ & 184.5 & $50-500$ & 287.8 \\
Operative time (minute) & $45-120$ & 75 & $90-210$ & 148.8 \\
Average hospital stay (days) & $6-\mathrm{Mar}$ & 4.2 & $3-\mathrm{Feb}$ & 2.3 \\
Follow up (months) & 24-Apr & 13 & I8-Mar & 12 \\
\hline
\end{tabular}




\section{Discussion}

In managing patients presenting with ovarian dermoid cysts, the authors' approach of choice prioritized avoiding spillage of the cyst content into the peritoneal cavity. With this mindset, the vigilant attempts were rewarded with an outstanding outcome as results shown not a single incident of cyst rupture during excision. In our opinion, surgical excision is superlative to other methods of treatment. When surgery is decided, it should be executed with even more caution than usual not to cause spillage of cyst content to the abdominal cavity or surrounding anatomical structures, with most attention paid if the cyst was infected or showed evidence of necrosis or suspected to be a malignant one. Spillage of cyst content into the abdominal or pelvic cavity has the potential to cause serious complications such as foreign body reaction, chemical peritonitis or the avoidable peritoneal dissemination of malignancy. The presence of ovarian cysts is considered a natural phenomenon. In other words, ovarian cyst can be classified into two groups, the functional group and the complex one. As per their designated name, functional cysts fulfill a certain role of ovarian functions, for example they play a part in the release of ovum at the appropriate time for fertilization. In contrast to complex cysts which do nothing of the sort.

Despite containing mature tissue such as skin complete with sweat glands, hair follicles as well as pockets of blood, fat, bone, cartilage, nails, teeth and even traces of thyroid tissue dermoids don't carry any functional value or benefit, quite the contrary, their presence can cause harm to the ovarian tissue and further long term complications. ${ }^{14}$ Although Dermoids in general portray a very benign behavior that doesn't call for alarm, serious complications are possible if not detected in due time. Both ovarian cysts and dermoid ovarian tumors can give rise to similar symptoms and for such symptoms both entities are on top of the list for the differential diagnosis. Symptoms may be exaggerated or become more severe when the cyst expands in size and exerts pressure on the ovary or other pelvic organs. In certain situations, a dermoid ovarian tumor may lead the ovary to rotate upon the axis of its blood supply and cut it off, causing ischemia and pain. Not all women experience symptoms in the same severity or intensity. Some percentage of women will be asymptomatic of a dermoid ovarian cyst, while most will encounter symptoms varying from severe to mild in intensity. In our study, 16 patients $(18.39 \%)$ were incidentally found to have dermoid ovarian cysts, while abdominal mass was described by $35(40.22 \%)$ of patients, on the other hand, some form of pain described as either dull continuous pain or sharp shooting pain and in 11 patients (12.64\%) reported the pain to be during sexual activity. Others reported abnormality in the menstrual cycle which in turn was related to the dermoid. It is a well-known fact that dermoid ovarian cysts may be asymptomatic and only discovered as incidental finding when performing standard pelvic examination in the form of adnexal enlargement or swelling of the ovary. ${ }^{15}$ And it should be kept in mind that is well established as a fact in gynecological science the prevalence of malignant transformation in dermoid cysts has been reported in $1-3 \%$ of cases. ${ }^{16}$

When abnormal cysts grow in size, their chance of causing complications, with twisting the ovary being the most serious and in severe cases cutting off the blood supply causing necrosis in the ovarian tissue and irreversible damage and effect on future fertility prospects. In addition to the previously mentioned risk of ovarian torsion, there is an additional risk of cyst rupture and spillage of the content into the peritoneal cavity. In our study no incidents of rupture or spillage on the contents are reported despite the fact that there were 5 patients which had torsion ranging from 2 to 5 times increasing the risk of rupture and spillage. When managing patients, a holistic approach in which the patients is treated as whole, aiming to pinpoint the root cause and eliminating them. Once the source of the problem is dealt with the cyst goes away as does the risk of recurrence. Consequently, this holistic approach has had greater success rate in the management of all kinds of ovarian cysts. Open surgical excision when done skillfully is the most suitable method for complete excision of the cyst without spillage and keeping it intact. It is well known that the rate of spillage is reported to be $4-12 \%$ during laparotomy ovarian cystectomy. ${ }^{17,18}$ Nevertheless, in this study no incidence of spillage or rupture were reported as laparotomy ovarian cystectomy was coupled with skillful management of the cyst and fine surgical manipulation during surgery. Spillage of contents of the ovarian cyst especially in cases where there is a suspicion of malignancy persists as source for concern. The reported spillage rate via laparoscopic ovarian cystectomy is $15-100 \%,{ }^{19-21}$ in turn this spillage subjects the patient for further complications in the future ranging from adhesions formation and chronic pelvic pain and alterations in future fertility. A case of intraperitoneal dissemination in a patient who underwent laparoscopic cystectomy for a dermoid ovarian cyst that has undergone malignant transformation was reported in literature, in addition other reported cases of chemical peritonitis following rupture of a dermoid cyst during laparoscopic cystectomy. ${ }^{22,23}$ When the diagnosis is established, the surgeon is in an unenviable position, as there are many factors that direct and suggest the most appropriate surgical approach; laparoscopy versus laparotomy, cystectomy versus oophorectomy, taking into account; the experience of the surgeon in such procedures, many surrounding conditions such as the patient's surgical history, the presence of chronic diseases, others associated pathology, the facilities of the hospital, but, the biggest concern to exteriorize a dermoid cyst in laparoscopy with great carefulness of shattering the cyst and spillage of the contents into the peritoneal cavity together with another concomitant findings or the plan for another surgical procedures always addressed this concern; the laparoscopic approach theory; rupturing the cyst, aspiration the contents, cauterization the adjacent ovarian tissues, should be eradicated by being aware of its side effects to stop using it.

Vector Benezra et al. ${ }^{24}$ reported regarding the incidence of spillage based surgical approach adopted, comparing laparoscopy vs laparotomy for excision of dermoid ovarian cysts and found that the laparoscopic procedures; cystectomy versus oophorectomy having more spillage in the laparoscopic group $(66.7 \%$ versus $5.6 \%$ for cystectomy, $27.2 \%$ versus $5.6 \%$ in the oophorectomy group) than that in the laparotomy group. ${ }^{24}$ Keeping in mind that the laparoscopic approach for ovarian cystectomy is safe and of benefit when done by an experienced laparoscopic surgeon..$^{25,26}$ Conscious serious efforts should be paid towards an outlook to the future fertility of the patient and should not be limited to the narrower perspective of possible malignancy. The advancement in the clinical implementation of laparoscopic surgery in recent years should not be glossed over. ${ }^{27.28}$ The dependence of some on the use of assisted reproductive methods as an alternative is not a justification for providing treatment facilities; because our social and cultural habits sanctify the childbearing, and most families cannot provide the necessary funds for this procedure, be that as it may, there should be a specific guidelines governing its application, as each case is individualized and each institute has its working circumstances and available experience. It must be emphasized that trials are being undertaken to have some criteria for selected cases of ovarian dermoid cysts to be excised laparoscopically. Still that does not come without its restraints due to the fact that the status of the setup and equipment in the hospital is not contemporary, thus limiting the ability to undertake this type of surgery. However, prominent skills were obtained through training abroad. It is Worthy of 
noting that a main success factor of this study lied in the collaboration, coordination and mutual exchange of data by, between and amongst the authors of this study.

\section{Conclusion}

For the ultimate purpose of preserving future fertility and ovarian functionality, the management of young patients presenting with dermoid ovarian cyst, we believe it should be via skillful laparotomy ovarian cystectomy. Despite their significance when present and the health risks they pose to women when present, ovarian cysts are not typically at the forefront of one's mind. Their absence from one's mind is reflected in delay in diagnosis and therefore increases in risk of complications. Laparoscopic approach seemed to be an ideal, attractive, and applicable method for small sized adnexal masses with optimal operative circumstances and well-trained personnel's. A way for reducing this risk is by performing a thorough physical examination coupled with imaging evaluation. The fact that the presence of ovarian dermoid cysts may be reflected in the form of ovarian enlargement, further stresses the importance of regular checkups and the need for implementing a screening program in high risk population. Such programs should be given priority in the national health care policy strategies in the future. But such strategies need large perspective controlled studies to compare conventional methods with laparoscopy in cases with dermoid and to assess rarer events like undetected malignancy, chemical peritonitis or excess adhesion formation. Focusing to gain the laparoscopic surgical skills may be the suitable gate for modern surgery if designed properly.

\section{Acknowledgments}

The authors would like to thank all the patients for their willingness to participate in the study and their patience.

\section{Funding}

We received no funding or benefits from any source or establishment to conduct our study.

Source(s) of support: Jordan University Hospital (In-kind).

\section{Conflicts of interest}

The authors declare no conflicts of interest in preparing this article.

\section{References}

1. Qurieno D, Ghaneh F, Michel C, et al. Benign rapidly growing ovarian dermoid cysts: a case series. Journal of Diagnostic Medical Sonography. 2016;33(1):71-74.

2. Kathleen EO, Amber RC. The approach to ovarian dermoids in adolescents and young women. J Pediatr Adolesc Gynecol. 2011;24(3):176-180.

3. Li RY, Nikam Y, Kapurubandara S. Spontaneously ruptures dermoid cysts and their potential complications: A review of literature with a case report. Case Rep Obstet Gynecol. 2020;2020:6591280.

4. Khanna, S, Srivastava, V, Saroj, S, et al. An unusual presentation of ovarian teratoma: a case report. Case Rep Emerg Med. 2012;845198.

5. Mishra TS, Jena SK, Kumari S. et al. Cecal dermoid masquerading dermoid cyst of ovary: a case report and review of the literature. $J$ Med Case Reports. 2021;15:38.

6. Wolter Oosterhuis J, Leendert H Looijenga. Human germ cell tumours from a developmental perspective. Nature Reviews Cancer. 2021;19:522_537.
7. Yuen PM, Yu KM, Yip SK, et al. A randomized prospective study of laparoscopy and laparotomy in the management of benign ovarian masses. Am J Obstet Gynecol. 1997;177(1):109-114.

8. Ozgur T, Atik E, Silfeler DB, et al. Mature cystic teratomas in our series with review of the literature and retrospective analysis. Arch Gynecol Obstet. 2013:1099-1101.

9. Kurt S, Avşar HA, Doğan ÖE, et al. Effects of mature cystic teratoma on reproductive health and malignant transformation: A retrospective analysis of 80 cases. J Turk Ger Gynecol Assoc. 2019;20(2):84-88.

10. Wang C, Thia E, Lim T. An adnexal mass with raised serum beta-HCG: an unusual presentation of ovarian dermoid cyst. Eur J Obstet Gynecol Reprod Biol. 2012;160(2)238-239.

11. An-Jen Chiang, Min-Yu Chen, Chia- Sui Weng, et al. Malignant transformation of ovarian mature cystic teratoma into squamous cell carcinoma: a Taiwanese Gynecologic Oncology Group (TGOG) study. $J$ Gynecol Oncol. 2017;28(5):e69.

12. Black JD, Roque DM, Pasternak MC. A series of malignant ovarian cancers arising from within a mature cystic teratoma: a single institution experience. Int J Gynecol Cancer. 2015;25(5):792-797.

13. Nazan Deniz Yön, Cansu Akbulut. Identification of primordial germ cells: cytological, histological and immunohistochemical aspects. Braz arch biol technol. 2015;58(2).

14. Rebecca Yuan Li, Yogesh Nikam, Supuni Kapurubandara. Spontaneously ruptured dermoid cysts and their potential complications: a review of the literature with a case report. Case Reports in Obstetrics and Gynecology. 2020 .

15. Azami A, Iliass E, Abdeerrahman S, et al. An unusual presentation of ovarian dermoid cyst: A case report and review of literature. Obstetrics and Gynecology Science. 2018;61(4):529.

16. Manju Rachel Mathew, Anita Ramdas, Susy S Kurian, et al. A rare malignant transformation of an ovarian cystic teratoma: a case report. Case Reports in Pathology. 2018.

17. Jaworski RC, Boadle R, Greg J, et al. Peritoneal melanosis associated with a ruptured ovarian dermoid cyst: report of a case with electron-probe energy dispersive X-ray analysis. Int J Gynecol Pathol. 2001;20(4):386389 .

18. Godara R, Karwasra RK, Garg P, et al. A diagnostic symptom of ovarian dermoid cyst. Ultrasound Obstet Gynecol. 2010;35:751-753.

19. Nishida M, Kawano Y, Yuge A, et al. Three cases of immature teratoma diagnosed after laparoscopic operation. Clin Med Insights Case Rep. 2014.

20. Park SB, Kim JK, Kim KR, et al. Imaging findings of complications and unusual manifestations of ovarian teratomas. Radio Graphics. 2008;28(4):969-983.

21. Tejani AS, He L, Zheng W, et al. Concurrent presentation of immature ovarian teratomas with refractory hyponatremia. A case report. J Clin Imaging Sci. 2020;10:23.

22. Bužinskienė D, Mongirdas M, Mikènas S, et al. Chemical peritonitis resulting from spontaneous rupture of a mature ovarian cystic teratoma: a case report. Acta Med Litu. 2019;26(4):217-226.

23. Laura E Eisman, Carolyn Stull, Larry I Barmat. Prolonged chemical peritonitis following intraperitoneal rupture of a dermoid cyst. Journal of Gynecologic Surgery. 2017:1-3.

24. Victor B, Usha V, R Wayne Whitted. Comparison of laparoscopy versus laparotomy for the surgical treatment of ovarian dermoid cysts. Gynecological surgery. 2005;2:89-92.

25. Berek J, Hacker F. Berek \& Hacker gynecologic oncology. 5th Edition 2010:455-459. 
26. Krista I, Childress J, Gisselle PM, et al. Intraoperative rupture of ovarian dermoid cysts in the pediatric and adolescent population: should this change your surgical management? J Pediatr Adolesc Gynecol. 2017;30(6):636-640.

27. Kamil MF, Shawqi SS, Farah KF, et al. Setting priorities in the conventional approaches in managing ectopic pregnancy; is it time to reform? A teaching hospital experience. Menopause Review. 2020;18(4):1-5.
28. Kamil M Fram, Asma S Basha, Farah K Fram, et al. Approach of gynecological cancers treated at Jordan University Hospital: Facts and Figures. European journal of gynaecological oncology. 2020:89-92. 